\title{
Differential Diagnosis of Hallucinatory Experiences in the Elderly
}

Afonso, H. ${ }^{1}$; Froes, V.1; Leandro, F.; Varela, I. ${ }^{1}$; Esteves, S. ${ }^{1}$; Carneiro, M. ${ }^{1}$; Lima, G; Martins, J. ${ }^{2}$

1 - Department of Psychiatry and Mental Health, Centro Hospitalar do Barreiro Montijo, Barreiro, Portugal; 2 - Department of Psychiatry and Mental Health, Distrital Hospital of Santarém, Santarém, Portugal

\section{Background/Aims}

\section{- Hallucinations are perceptions without an object with the strength of a real perception;}

- Are experienced as normal sensorial experiences and may happen in psychiatric and medical patients and healthy individuals; In non-clinical populations, isolated hallucinations are highly frequent reaching rates of even $71 \%$;

- Some authors question the specificity of hallucinatory events and their meaningfulness as equivalents of psychiatric illness classifying them in a continuum or range of severity

- Normality or pathology determined by frequency, content, subjective distress, degree of insight and evidence of physical or mental disorder;

- In elderly, hallucinatory experiences may occur in multiple disorders: demential syndromes, depressive disorders, grief, sensorial acuity decrease, delirium, substance effects, neurological lesions, Parkinson Disease, late onset schizophrenia, bipolar disorder and post-traumatic stress disorder.

\section{Objectives}

With this work the authors want to review the differential diagnosis of hallucinatory symptoms in the elderly.

\section{Materials and methods}

The authors reviewed, non systematically, the literature, through search in textbooks and papers related to this topic in the databases of Pubmed and Sciencedirect.

\section{Results}

Important the phenomenological differentiation of these perceptive experiences with other false perceptions - illusions and pseudoallucinations:

- Illusion - transformation of a perception by mixture of a fantasious perceptive experience with a normal perception;

- Pseudo-hallucinations - distinguished either by insight revealed by the patient (hallucination - without insight; pseudo-hallucination with insight) or location where the phenomena takes place (hallucination outside the internal space; pseudo-hallucination inside the internal space).

Next, are summarized the most common hallucinatory experiences in elderly categorized by type of hallucination with differential diagnosis:

- Commonly secondary to organic causes;

\section{Visual Hallucinations}

- Appear in oftalmologic, neurologic, metabolic, iatrogenic or psychiatric disorders;

- Elementary to complex

- May happen in intoxication or abstinence of substances - investigate temporal relationship;

- Linked to multiple diagnosis: retinal pathology; cortical blindness; migraine; occipital lobe epilepsy and tumors; Parkinson Disease (simple or complex people, animals); Lewy Body Dementia (important for diagnosis - fluctuating); other dementia causes - with "sundowning effect" (more on evening and night); cortical blindness; delirium (>25\% patients); post-concussional; hepatic disorders; Charles Bonnet Syndrome (visual acuity); delirium tremens; autoscopic phenomena (depressive syndromes).

- Healthy individuals or medical or psychiatric patients;

- Elementary to complex

- Linked to: dementia; delirium; substance induced psychosis; sensorial deficit caused hallucinations; psychiatric disorders

- Psychiatric disorders: late onset or very late onset schizophrenia (more in women); persistent delusional disorder (related to delusions and not significative in the clinical picture); depressive or bipolar disorder with psychotic componente; less data on some disorders (OCD; anxious disorders; dissociative disorders; personality disorders)

- Neurological disorders: dementia (Alzheimer, Parkinson; Lewy body); migraine; neurosyphilis; temporal lobe epilepsy; encephalopaty; focal brain lesions; narcolepsy; Huntington disorder; eritematous systemic lupus; Hashimoto encephalopaty.

- Other causes; auditive sensorial deficits; legal and ilegal substance effect; delirium; auditive interference on ear aids; ear pathology; major acute stressors; sleep related hallucinations

- Related to abnormal bodily sensations;

Cenestesic hallucinations

- Described as related to mood disorders, delusional disorders, dementia, schizophrenia, neurological causes as stroke;

- Also related to pharmacological treatments - dopaminergic agonists.

- Described in the context of:

Olfatory hallucinations

- delusional disorder; dementia; epilepsy; tumors of temporal lobe.

\section{Conclusions}

- Differential diagnosis of hallucinatory symptoms in the elderly present a serious challenge;

- May be related to multiple medical and psychiatric causes and occur with multiple comorbidity in the elderly;

- Difficulty due to inespecificity of the hallucinatory symptoms;

- Important to achieve an accurate diagnosis in order to elicit the appropriate treatment. 